\title{
An Energy Efficient and Cooperative Congestion Control Protocol in MANET
}

\author{
Md. Imran Chowdhury \\ B.Sc. in CSE \\ Department of CSE, CUET \\ Chittagong-4349, Bangladesh
}

\author{
Asaduzzaman, $\mathrm{PhD}$ \\ Assistant Professor \\ Department of CSE, CUET \\ Chittagong-4349, Bangladesh
}

\author{
Mir. Md. Saki Kowsar \\ Assistant Professor \\ Department of CSE, CUET \\ Chittagong-4349, Bangladesh
}

\begin{abstract}
ABSTRAC
This paper presents an energy efficient and cooperative congestion control protocol to control the congestion in mobile adhoc networks (MANETs). The proposed scheme overcomes the disadvantages of existing multicast congestion control protocols which depend on individual receivers to detect congestion and adjust their receiving rates. In the first phase of the proposed protocol, it builds a cooperative multicast tree rooted at the source, by including the nodes with higher residual energy towards the receivers. In the second phase of the proposed protocol, it proposes an admission control scheme in which a cooperative multicast flow is admitted or rejected depending upon on the output queue size. In the third phase of the proposed protocol, it proposes a scheme which tests whether the relay node has the potential path to the required destination, if not then choose the another node which has the second most highest residual energy as a new relay node. That is more generally introduction of cooperativeness and making it. In the fourth phase, we propose a scheme which adjusts the multicast traffic rate at each bottleneck of a multicast tree. Because of the on-the-spot information collection and rate control, this scheme has very limited control traffic overhead and delay. Moreover, the proposed scheme does not impose any significant changes on the queuing, scheduling or forwarding policies of existing networks. Simulation results shows that the proposed protocol has better delivery ratio and throughput with less delay and energy consumption when compared with existing protocol.
\end{abstract}

Keywords- MANET, Congestion, AODV, EERCCP, Multicasting, Cooperative transmission.

\section{INTRODUCTION}

A mobile ad-hoc network (MANET) is composed of mobile nodes without any infrastructure. The goal of MANETs is to extend mobility into the realm of autonomous, mobile and wireless domains, where a set of nodes form the network routing infrastructure in an ad-hoc fashion. The majority of applications of MANETs are in areas where rapid deployment and dynamic reconfiguration are necessary and wired network is not available. These include military battlefields, emergency search, rescue sites, classrooms and conventions, where participants share information dynamically using their mobile devices. These applications lend themselves well to multicast operations. [1]. Due to mobility of nodes in MANET, it is not possible to establish fixed paths for message delivery through the network. Hence, congestion is happening and it is the key problem for MANET. Actually,
Congestion is situation in communication network when too much traffic is offered and the Congestion on MANET leads to Packet losses, Bandwidth degradation, Wasting of time (i.e. long delay), High overhead. So, many routing protocols have been proposed to overcome the congestion in MANET.

One of the popular routing protocol is AODV, which is used to send the messages over MANET and also to overcome the congestion in MANET. But, it depends on individual receivers to detect congestion and adjust their receiving rates. Another common routing protocol is EERCCP, which is better than AODV but sometimes it fails when relay node gets in trouble cause there is no other mechanism to select an alternative relay nodes i.e. Lack of cooperativeness. Hence, we proposed a routing protocol named as EECCCP. It encorporates the benefits of energy efficiency and cooperativeness which in turn reduces the congestion effectively.

The proposed energy efficient and cooperative congestion control routing protocol (EECCCP) will perform well better than both the AODV and EERCCP. Moreover, the proposed scheme does not impose any significant changes on the queuing, scheduling or forwarding policies of existing network protocols. Simulation results have shown that the proposed protocol EECCCP has better delivery ratio and throughput with less delay and energy consumption when compared with existing protocol AODV and EERCCP.

\section{RELATED WORK}

In [3] Long Cheng, Sajal K.Das, Canfeng Chen, Jian Ma and Wendong Wang, proposed an approach which is named as "Robust Forwarding for Reactive Routing Protocols in wireless ad hoc networks with Unreliable Links," IEEE ICC. The key idea of their proposed scheme is to get the better result than AODV. To achieve the better result they have taken the benefits of cooperativeness. But, their proposed scheme is not energy efficient because each guide node requires more energy to maintain the cooperativeness among them. The relation of this approach to our proposed protocol is to control the congestion with the help of cooperativeness in MANET.

Hua Chen, Baolin Sun [4] introduces an Entropy-based Fuzzy controllers QoS Routing algorithm in MANET (EFQRM). The key idea of EFQRM algorithm is to construct the new metric-entropy and fuzzy controllers with the help of entropy metric to reduce the number of route reconstruction so as to provide QoS guarantee in the ad hoc network. But, due to lack of cooperativeness it fails sometimes to construct the new 
metric-entropy and fuzzy controllers. The relation of this approach to our proposed protocol is they worked on MANET and tried to provide the QoS guarantee in MANET.

In [5] Dr.G. Sasi Bhushana Rao, M.RajanBabu, proposed an approach which is "An Energy Efficient and Reliable Congestion Control Protocol for Multicasting in MANET," abbreviated as EERCCP which provides better result than AODV. Their proposed protocol is energy efficient. But, it fails when link failure happens to relay nodes or when relay node moves its current group to another group i.e. it has the lack of cooperativeness. The relation of this approach to our proposed protocol is to control the congestion with the help of energy efficiency in MANET.

\section{PROPOSED PROTOCOL}

\subsection{Overview}

This paper presents an energy efficient and Cooperative congestion control protocol for cooperative multicasting in mobile ad hoc networks (MANETs). The proposed scheme overcomes the disadvantages of existing multicast congestion control protocols which depend on individual receivers to detect congestion and adjust their receiving rates such as AODV. Since, congestion is the key problem for MANET. Hence, to control the congestion and also to overcome the disadvantages of existing multicast congestion control protocol we proposed a protocol. It encorporates the benefits of energy efficiency and cooperation-based routing algorithm, namely, an energy efficient and cooperative congestion control protocol (EECCCP), which makes full use of the cooperative communications while constructing the minimum-power route. Moreover, the proposed scheme does not impose any significant changes on the queuing, scheduling or forwarding policies of existing network protocols. Simulation results have shown that the proposed protocol EECCCP has better delivery ratio and throughput with less delay and energy consumption when compared with existing protocol AODV and EERCCP which is proposed in [5].

\subsection{Cooperative Energy Efficient Tree \\ Construction}

Here, the proposed energy efficient and cooperative congestion control protocol builds a cooperative multicast tree rooted at the source towards the receivers. The distance i.e. the geographical location of the nodes is assumed. Their residual energy is measured. The nodes are sorted based on its location from the source and arranged in a sequence order. A distance threshold value $Q$ is set and the nodes which are less than $Q(n<Q)$ are multicast from the source and the nodes which are greater than $Q(n>Q)$ are multicast by the relay node where $n$ is the nodes. In case of multicasting the node which has the maximum residual energy per corresponding receiver is set as the relay node. The relay node then forwards the packets from the source to the corresponding receivers. But, since MANET topology is dynamic i.e. its nodes have the random motion hence if relay nodes fails to forward the packets to the receiver then the nodes having the second most highest residual energy must be chosen as a relay node for the multicasting purposes.
Calculating Residual Energy of a Node: Consider a network with multicast groups $G_{1}, G_{2}, \ldots \ldots \ldots \ldots \ldots, G_{x}$. Each group $\left\{G_{i}\right\}$ consists of $N$ nodes. Every node in the MANET calculates its remaining energy periodically. The nodes may operate in either transmission or reception mode. Let $\left\{E_{1}, E_{2}, \ldots \ldots \ldots, E_{n}\right\}$ are the residual energies of the nodes measured by the following method. The power consumed for transmitting a packet is given by

$$
\text { Consumed energy }=T_{p} * t
$$

Where $T_{p}$ is the transmitting power and $t$ is transmission time.

The power consumed for receiving a packet is given by

$$
\text { Consumed energy }=R_{p} * t
$$

Where $R_{p}$ is the reception power and $t$ is the reception time. The value $t$ can be calculated as $t=D_{s} / D_{r}$

Where $D_{s}$ is the Data size and $D_{r}$ is the Data rate.

Hence, the residual energy ( $E$ ) of each node can be calculated using (1) or (2) and (3)

$$
E=\text { Current energy }- \text { Consumed energy }
$$

Again, let us consider a cooperative multicast tree based on the above definition.

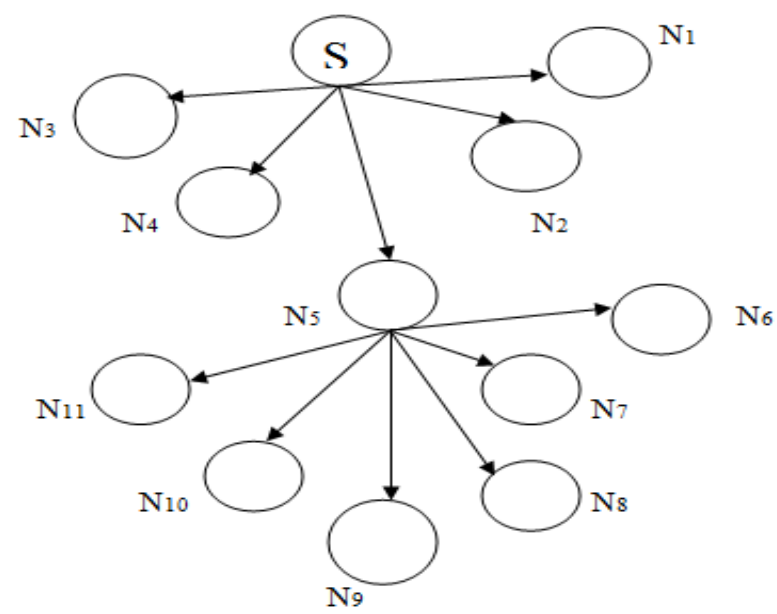

Figure 1: Cooperative Energy efficient tree construction 
Source $S$ multicast the packets to nodes $N_{1}, N_{2}, N_{3}, N_{4}$ and $N_{5}$ is the relay node. $N_{5}$ multicast the packets to the rest of the nodes $N_{6}, \ldots, N_{11}$. Now, let a link failure has happened to relay node $N_{5}$ or the relay node moved its current group to another group. Then our proposed scheme will choose an alternative relay node which has the second most highest residual energy within this same group. Let, $N_{11}$ is the new relay node instead of the old relay node $N_{5}$. Then the cooperative multicast tree will be as follows:-

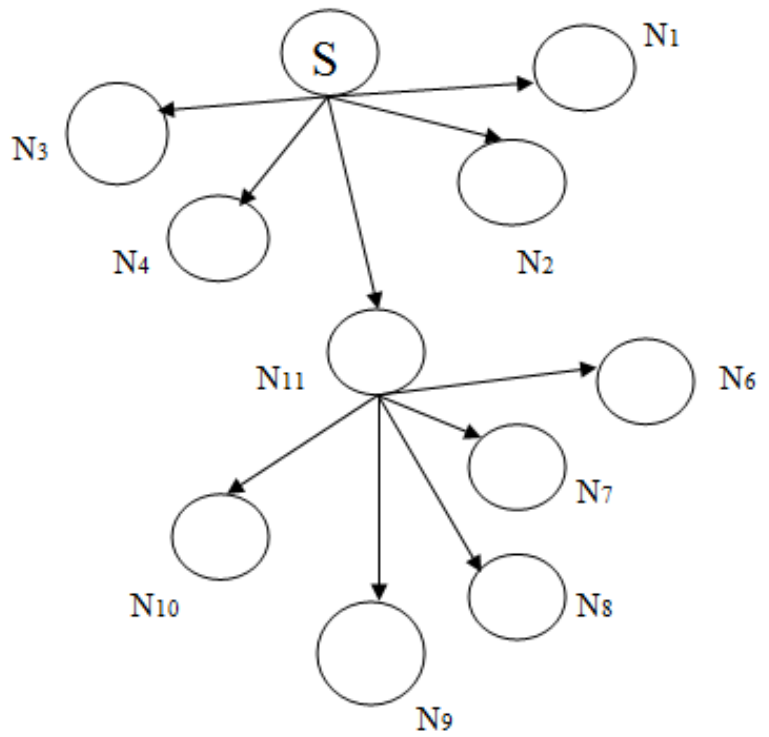

Figure 2: Cooperative Energy efficient tree construction

Source $S$ multicast the packets to nodes $N_{1}, N_{2}, N_{3}, N_{4}$ and $N_{11}$ is the new relay node. Then $N_{11}$ multicast the packets to the rest of the nodes $N_{6}, \ldots, N_{10}$.

\subsection{Algorithm for Proposed Protocol}

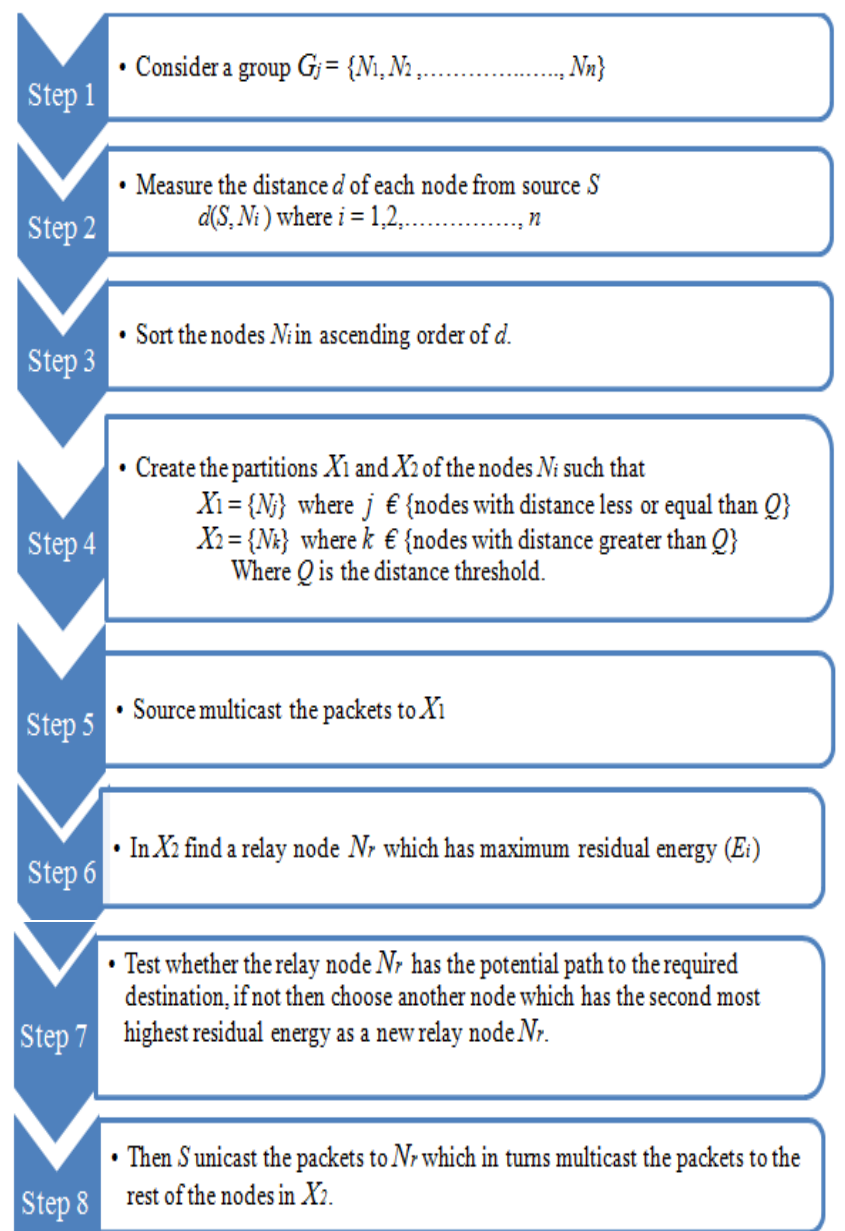

Figure 3: Algorithm for proposed protocol

\subsection{Cooperative Multicasting Admission Control}

Most of the existing schemes depend on individual receivers to detect congestion and adjust their receiving rates which are much disadvantageous. But the proposed protocol(EECCP), proposes a scheme which adjusts the multicast traffic rate at each bottleneck of a cooperative multicast tree. Each node estimates its current traffic load and arrival rate. Based on its traffic load, it estimates the receiving rate. If the receiving rate is less than the arrival rate, it adaptively adjusts its receiving rate. In order to adjust the total number of multicast flows which traverse a bottleneck, the following procedure is used. In our proposed scheme, based on the link's output queue state, multicast flows at a bottleneck can be blocked or released. Let the number of packets in the queue is $N$. Let $Q_{t 1}$ and $Q_{t 2}\left(Q_{t 1}<Q_{t 2}\right)$ are two thresholds for the queue size. Then the flow is released or blocked based on the following conditions.

$$
\begin{aligned}
& \text { If } N \leq Q_{t 1} \text {, then the multicast flow is released. } \\
& \text { If } N>Q_{t 2} \text {, then the multicast flow is blocked. }
\end{aligned}
$$


Whenever congestion happens or about to happens, then the multicast sessions which traverse the branch are blocked. Thus the packets are stopped from entering the branch. The blocked flows are released to traverse the branch when the branch is lightly utilized.

\subsection{Cooperative Multicasting Traffic Rate Adjustment}

When the available bandwidth is less than the required bandwidth, then it indicates the possibility of congestion or packet loss. In our proposed scheme, we increased the multicast traffic rate by a constant rate when congestion had not been happened. As well as we decreased the multicast traffic rate by a constant rate when the network was congested. The behavior of the multicast session is expressed as

$$
R(t+1)=\left\{\begin{array}{lc}
R(t)-g & \text { If } R(t)>B \\
R(t)+g & \text { If } R(t) \leq B \\
R(t) & \text {; otherwise }
\end{array}\right.
$$

Here, $R(t)$ denotes the instantaneous rate of the multicast session at time $t . B$ is the bottleneck bandwidth. When $R(t)>$ $B$ then the network is congested and the multicast session decreases its rate by a step $g$. Where $g$ is the $10 \%$ of $R(t)$. If $R(t) \leq B$ then the network is not congested and the multicast session increases its rate by a step $g$. Where $g$ is the $10 \%$ of $R(t)$.

\section{SIMULATION RESULTS}

We use NS2 to simulate our proposed protocol. In case of simulation, the channel capacity of mobile hosts is set to the same value: 2 Mbps. We use the distributed coordination function (DCF) of IEEE 802.11 for wireless LANs as the MAC layer protocol. It has the functionality to notify the network layer about link breakage. The simulation settings and parameters are summarized in the following table 1 .

\section{TABLE 1 : SIMULATION PARAAMETERS}

\begin{tabular}{|c|c|}
\hline Number of Nodes & 50 \\
\hline Area Size & $1000 \times 1000$ \\
\hline MAC & 802.11 (DCF) \\
\hline Radio Range & {$[50,100,150,200,250]$} \\
\hline Simulation Time & 1.5 minutes \\
\hline Traffic Source & CBR \\
\hline Packet Size & $250 \longleftarrow 650,700 \longleftarrow$ 1000 \\
\hline Node Density & {$[5,10,15,20,25,30,35,40,45,50]$} \\
\hline
\end{tabular}

\begin{tabular}{|c|c|}
\hline Mobility Model & Random way point \\
\hline Pause Time & $5 \mathrm{sec}$ \\
\hline Transmit Power & $31.32 \mathrm{e}-3$ \\
\hline Receiving Power & $35.28 \mathrm{e}-3$ \\
\hline Idle Power & $712 \mathrm{e}-6$ \\
\hline Speed & Random motion \\
\hline
\end{tabular}

\subsection{EVALUATION CRITERIA}

We compare the proposed EECCCP protocol with the multicast EERCCP [5] protocol and multicast AODV [6] protocol. We evaluate mainly the performance according to the following metrics.

\subsubsection{Average End-to-End Delay}

The lower value of end-to-end delay means the better performance of the protocol.

End to end delay $=\Sigma$ (arrive time - send time $)$

The end-to-end-delay is averaged over all surviving data packets from the sources to the destinations.

\subsubsection{Average Energy Consumption}

The power consumed for transmitting a packet is given by

$$
\text { Consumed energy }=T_{p} * t
$$

Where $T_{p}$ is the transmitting power and $t$ is transmission time. The power consumed for receiving a packet is given by

$$
\text { Consumed energy }=R_{p} * t
$$

Where $R_{p}$ is the reception power and $t$ is the reception time. Here, the average energy consumed by the nodes in receiving and sending the packets are measured.

\subsubsection{Average Packet Delivery Ratio}

It is the ratio of the No. of packets received successfully and the total no. of packets sent. Hence, the greater value of packet delivery ratio means better performance of the protocol. Following is the equation to calculate the packet delivery ratio. 


\subsubsection{Average Throughput}

It is defined as the total amount of data per time unit that is delivered from one node to another via a communication link. Or it is the number of packets received by all the nodes in the network. It can be calculated by the following equation:
From Figure 4 and Figure 5 we clearly see that the proposed protocol EECCCP gives the better result than the EERCCP and AODV.

Throughput $=\Sigma$ RecvdSize $/ \Sigma($ stopTime-startTime $)$

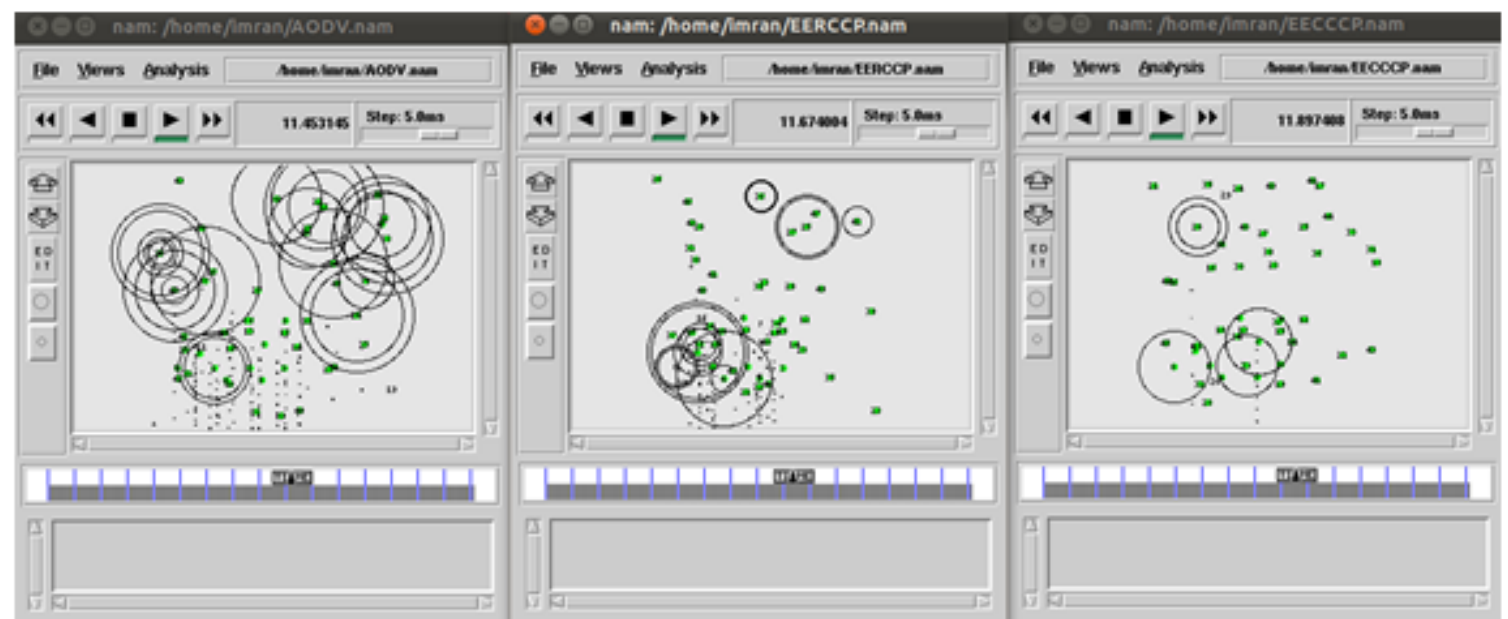

Figure 4: Comparison of EECCCP with respect to EERCCP and AODV

\subsection{RESULTS}

\subsubsection{Comparisons of simulation models}

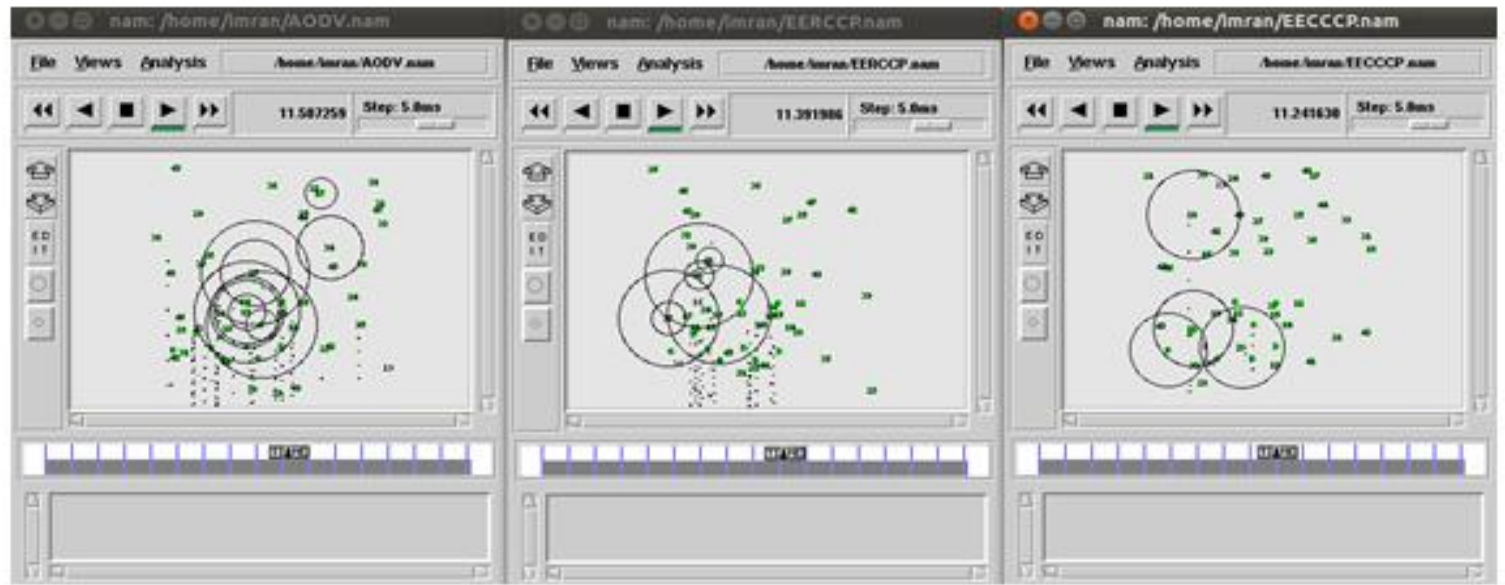

Figure 5: Comparison of EECCCP with respect to EERCCP and AODV

\subsubsection{Based on Node Density}

In this experiment, we vary the group size or the number of nodes per group as $5,10, \ldots ., 50$. 


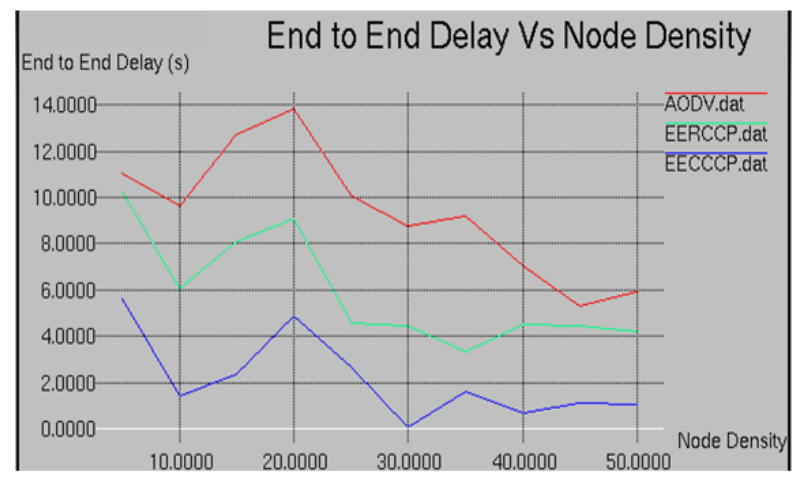

Figure 6: End-to-End Delay Vs Node Density on MANET

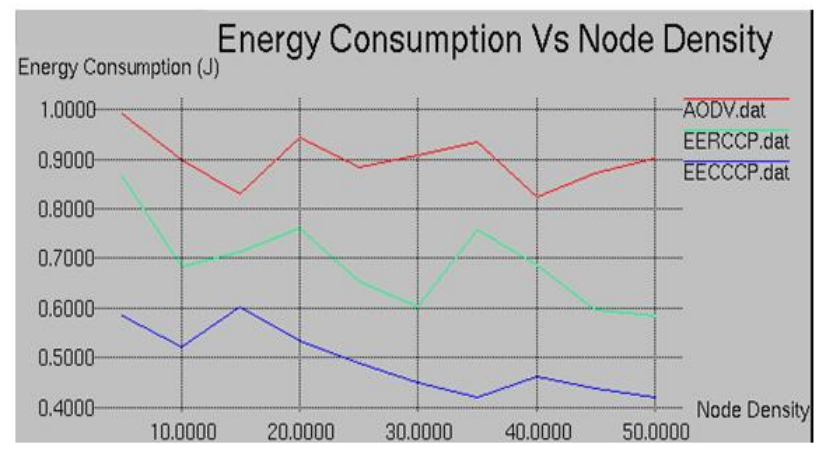

Figure 7: Energy Consumption Vs Node Density on MANET

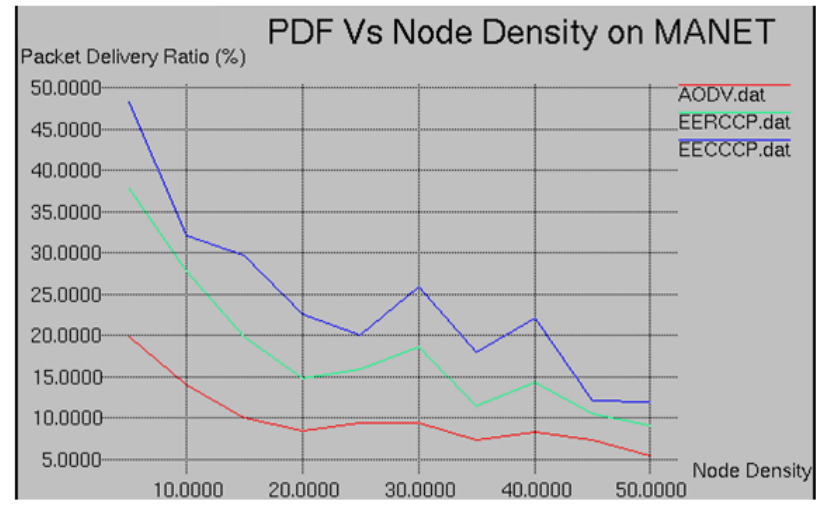

Figure 8: Packet Delivery Ratio Vs Node Density on MANET

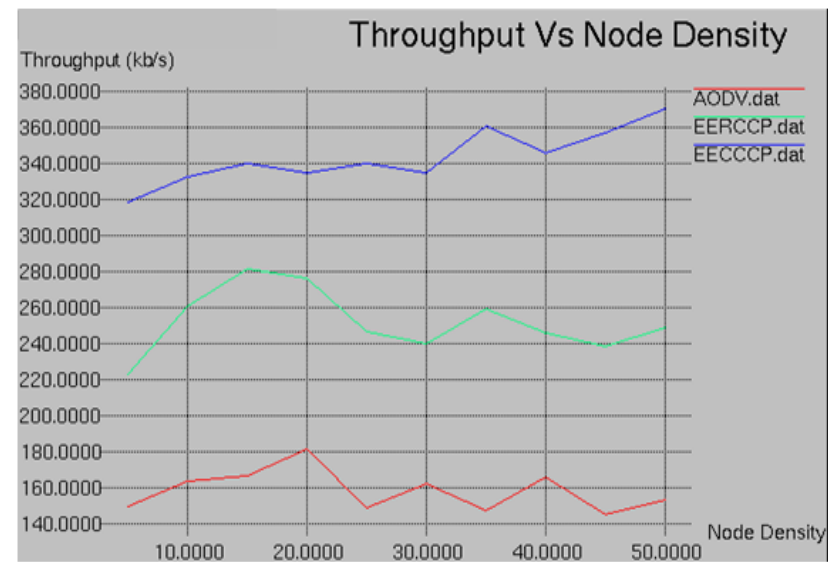

Figure 9: Throughput Vs Node Density on MANET

\section{When the number of nodes is increased:}

Figure 6 shows the average end-to-end delay occurred for the AODV, EERCCP and EECCCP.As we can see from the figure, the delay is less for Proposed Protocol EECCCP, when compared to AODV and EERCCP. Due to the benefits of cooperativeness to the proposed protocol EECCCP, it has the less delay than when compared to AODV and EERCCP.

Figure 7 shows the average energy consumption for all the cases. As we can see from the figure, the energy consumption is less for Proposed Protocol EECCCP, when compared to AODV and EERCCP. Due to the benefits of energy efficiency to the proposed protocol EECCCP, it has the less energy consumption than when compared to AODV and EERCCP.

Figure 8 shows the average delivery ratio for the AODV, EERCCP and EECCCP. As we can see from the figure, the delivery ratio is high for Proposed Protocol EECCCP, when compared to AODV and EERCCP. Due to the benefits of energy efficiency and cooperativeness to the proposed protocol EECCCP, it has the high packet delivery ratio than when compared to AODV and EERCCP.

Figure 9 shows the average throughput occurred for all the three case i.e. for the AODV, EERCCP and the Proposed Protocol EECCCP. As we can see from the figure, the throughput is high for Proposed Protocol EECCCP, when compared to AODV and EERCCP. Due to the benefits of energy efficiency and cooperativeness to the proposed protocol EECCCP, it has the high throughput than when compared to AODV and EERCCP.

\subsubsection{Based on Packet Size}

In this experiment, we vary the packet size as $250,300, \ldots \ldots \ldots, 500,550, \ldots \ldots . .950,1000$.

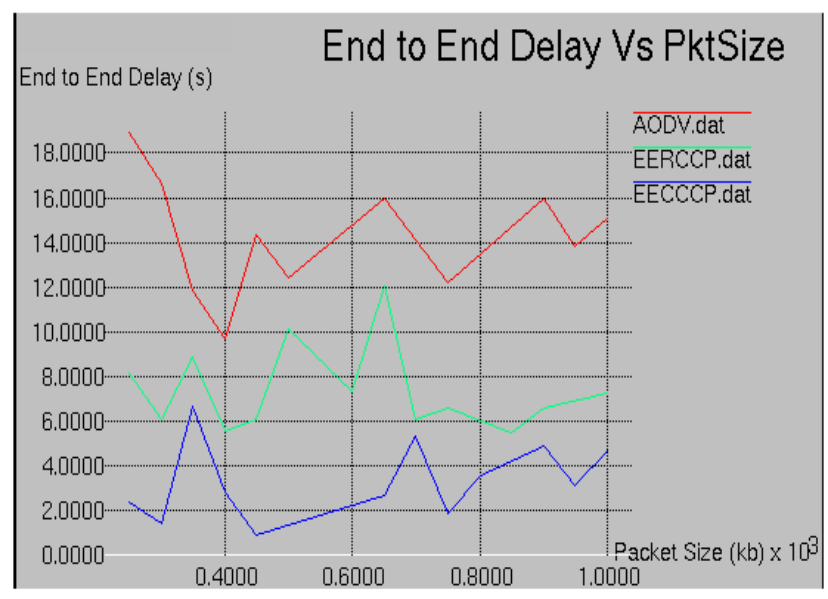

Figure 10: End-to-End Delay Vs Packet Size on MANET 


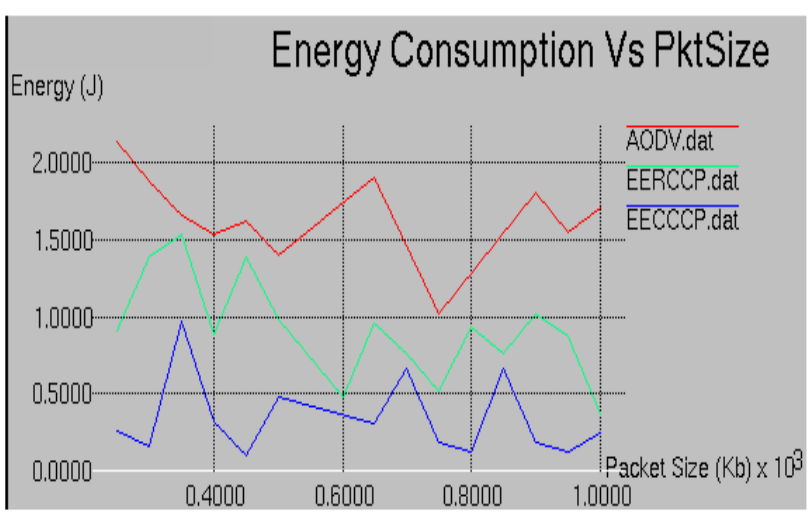

Figure 11: Energy Consumption Vs Packet Size on MANET

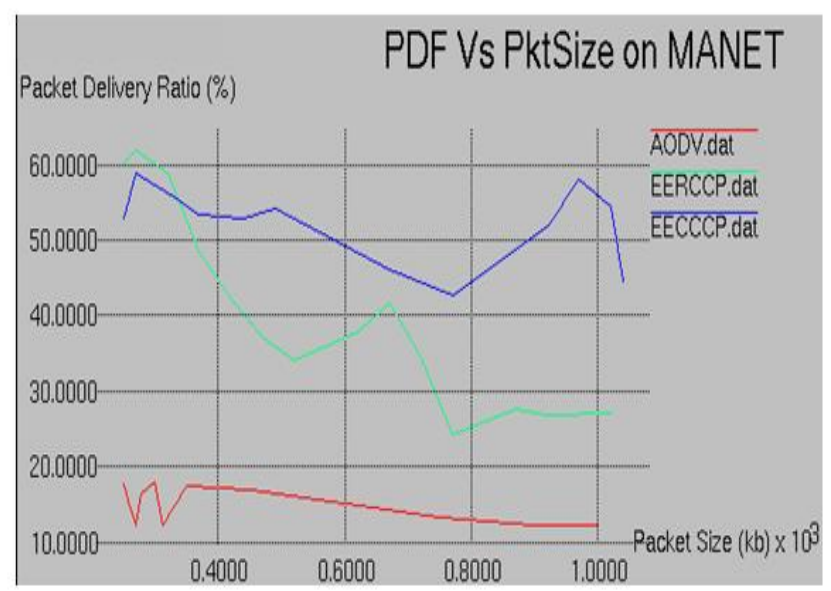

Figure 12: Packet Delivery Ratio Vs Packet Size on MANET

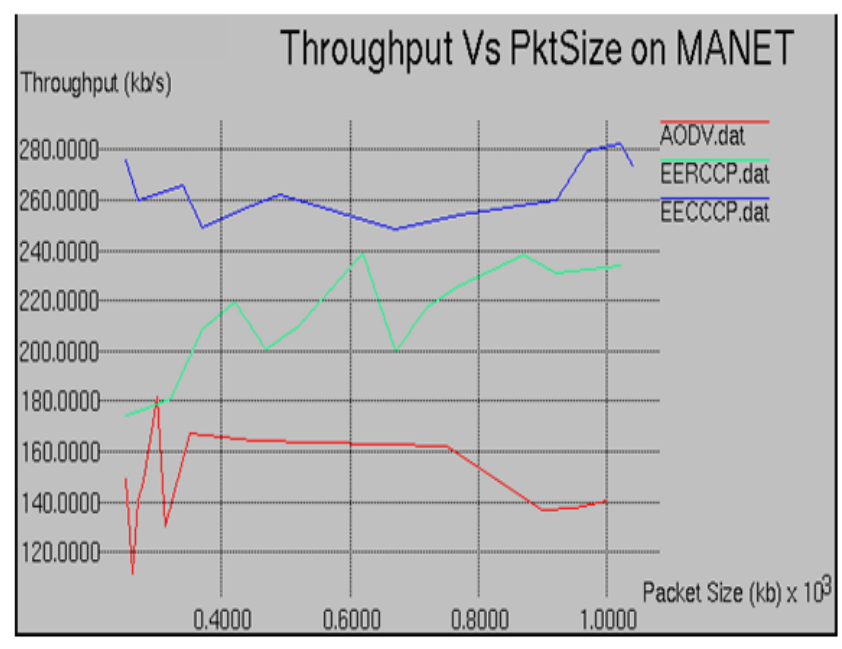

Figure 13: Packet Size Vs Throughput on MANET

\section{When the packets size is increased:}

Figure 10 shows the average end-to-end delay occurred for the AODV, EERCCP and EECCCP.As we can see from the Figure 10 shows the delay is less for Proposed Protocol EECCCP, when compared to AODV and EERCCP. Due to the benefits of cooperativeness to the proposed protocol EECCCP, it has the less delay than when compared to AODV and EERCCP.

Figure 11 shows the average energy consumption for all the cases. As we can see from the figure, the energy consumption is less for Proposed Protocol EECCCP, when compared to AODV and EERCCP. Due to the benefits of energy efficiency to the proposed protocol EECCCP, it has the less energy consumption than when compared to AODV and EERCCP.

Figure 12 shows the average delivery ratio for the AODV, EERCCP and EECCCP. As we can see from the figure, the delivery ratio is high for Proposed Protocol EECCCP, when compared to AODV and EERCCP. Due to the benefits of energy efficiency and cooperativeness to the proposed protocol EECCCP, it has the high packet delivery ratio than when compared to AODV and EERCCP.

Figure 13 shows the average throughput occurred for all the three case i.e. for the AODV, EERCCP and the Proposed Protocol EECCCP. As we can see from the figure, the throughput is high for Proposed Protocol EECCCP, when compared to AODV and EERCCP. Due to the benefits of energy efficiency and cooperativeness to the proposed protocol EECCCP, it has the high throughput than when compared to AOD and EERCCP.

\section{CONCLUSION}

In this paper, we have proposed an energy efficient and cooperative congestion control protocol (EECCCP) for cooperative multicasting in mobile ad hoc networks. The proposed protocol overcomes the disadvantages of existing multicast congestion control protocols such as AODV and EERCCP. The proposed protocol EECCCP, encorporates the benefits of energy efficiency and cooperativeness which in turn reduces the congestion effectively. Moreover, the proposed scheme does not impose any significant changes on the queuing, scheduling or forwarding policies of existing network protocols. Simulation results shows that the proposed protocol has better delivery ratio and throughput with less delay and energy consumption when compared with the existing network protocols.

This thesis work is closely related to the real life problems such as where rapid deployment and dynamic reconfiguration are necessary and wired network is not available. These include military battlefields, emergency search, rescue sites, classrooms and conventions, where participants share information dynamically using their mobile devices. These applications lend themselves well to multicast operations which is effectively done to the proposed protocol EECCCP, in this thesis work. 


\section{REFERENCES}

[1] Luo Junhai, Xue Liu, Ye Danxia," Research on multicast routing protocols for mobile ad-hoc networks", Elsevier, 2007.

[2] M. Sikora, J. N. Laneman, M. Haenggi, D. J. Costello, and T. E. Fuja, "Bandwidth- and power-efficient routing in linear wireless networks," IEEE Trans. Inform. Theory, vol. 52, pp. 2624-2633, June 2006.

[3] Long Cheng, Sajal K.Das, Canfeng Chen, Jian Ma and Wendong Wang, "Robust Forwarding for Reactive Routing Protocols in wireless ad hoc networks with Unreliable Links," IEEE ICC 2011 proceedings.

[4] Hua Chen, Baolin Sun introduces an Entropy-based Fuzzy controllers QoS Routing algorithm in MANET (EFQRM).
[5] Dr.G. Sasi Bhushana Rao, M.RajanBabu, "An Energy Efficient and Reliable Congestion Control Protocol for Multicasting in MANET," IJCSIS, vol.7,No. 1,2010.

[6] Elizabeth M. Royer, Charles E. Perkins," Multicast Operation of the Adhoc On-Demand Distance Vector Routing Protocol”,ACM,1999.

[7] F. Li, K. Wu, and A. Lippman, "Energy-efficient cooperative routing in multi-hop wireless ad hoc networks," in Proc. IEEE International Performance, Computing, and Communications Conference, pp. 215222, Apr. 2006

[8] J. N. Laneman, D. N. C. Tse, and G. W. Wornell Cooperative diversity in wireless networks: Efficient protocols and outage behavior. IEEE Transactions on Information Theory, 51(12):3062-3080, December 2004 